\title{
Shortened Acquisition Protocols for the Quantitative Assessment of the 2-Tissue-Compartment Model Using Dynamic PET/CT ${ }^{18}$ F-FDG Studies
}

\author{
Ludwig G. Strauss ${ }^{1}$, Leyun Pan ${ }^{1}$, Caixia Cheng ${ }^{1}$, Uwe Haberkorn ${ }^{1,2}$, and Antonia Dimitrakopoulou-Strauss ${ }^{1}$ \\ ${ }^{I}$ Clinical Cooperation Unit Nuclear Medicine, German Cancer Research Center, Heidelberg, Germany; and ${ }^{2}$ Department of Nuclear \\ Medicine, Ruprecht-Karls-University, Heidelberg, Germany
}

\begin{abstract}
${ }^{18}$ F-FDG kinetics are quantified by a 2-tissue-compartment model. The routine use of dynamic PET is limited because of this modality's 1-h acquisition time. We evaluated shortened acquisition protocols up to $0-30$ min regarding the accuracy for data analysis with the 2-tissue-compartment model. Methods: Full dynamic series for 0-60 min were analyzed using a 2-tissue-compartment model. The time-activity curves and the resulting parameters for the model were stored in a database. Shortened acquisition data were generated from the database using the following time intervals: $0-10,0-16,0-20,0-25$, and 0-30 min. Furthermore, the impact of adding a 60-min uptake value to the dynamic series was evaluated. The datasets were analyzed using dedicated software to predict the results of the full dynamic series. The software is based on a modified support vector machines (SVM) algorithm and predicts the compartment parameters of the full dynamic series. Results: The SVM-based software provides user-independent results and was accurate at predicting the compartment parameters of the full dynamic series. If a squared correlation coefficient of 0.8 (corresponding to $80 \%$ explained variance of the data) was used as a limit, a shortened acquisition of 0-16 min was accurate at predicting the 60-min 2-tissue-compartment parameters. If a limit of 0.9 (90\% explained variance) was used, a dynamic series of at least 0-20 min together with the 60-min uptake values is required. Conclusion: Shortened acquisition protocols can be used to predict the parameters of the 2-tissue-compartment model. Either a dynamic PET series of 0-16 min or a combination of a dynamic $\mathrm{PET} / \mathrm{CT}$ series of $0-20 \mathrm{~min}$ and a 60 -min uptake value is accurate for analysis with a 2-tissue-compartment model.
\end{abstract}

Key Words: PET; ${ }^{18} \mathrm{~F}-\mathrm{FDG}$; compartment modeling; quantification

J Nucl Med 2011; 52:379-385

DOI: 10.2967/jnumed.110.079798

$\mathbf{T}$ he standard radiopharmaceutical for PET examinations in oncologic patients is ${ }^{18} \mathrm{~F}$-FDG, a marker of tumor via-

\footnotetext{
Received Jun. 2, 2010; revision accepted Dec. 13, 2010.

For correspondence or reprints contact: Ludwig G. Strauss, Medical PET Group-Biological Imaging (E060-1), Clinical Cooperation Unit Nuclear Medicine, German Cancer Research Center, Im Neuenheimer Feld 280, D-69120 Heidelberg, Germany.

E-mail: Igs@ads-Igs.de

COPYRIGHT @ 2011 by the Society of Nuclear Medicine, Inc.
}

bility, which has been used with PET for many years now (1). The basic quantitative assessment of the tracer uptake is usually calculated using standardized uptake values (SUVs) - a method introduced by our group more than $19 \mathrm{y}$ ago as a ratio of the local tracer concentration with the injected dose and body volume (2). SUV is a distribution value, which is equal to 1 for a homogeneous distribution of the tracer and exceeds 1 if retention occurs in the tissue. The SUV or, alternatively, the maximum SUV in a volume of interest (VOI) has been used in many publications and was found to be useful as an additional parameter for tumor diagnosis and assessment of therapeutic effects. However, the SUV reflects the global uptake of a tracer and is dependent not only on the specific retention of ${ }^{18} \mathrm{~F}-\mathrm{FDG}$ but also on the fractional blood volume and other parameters (3). More detailed information can be obtained by compartment modeling. Vriens et al. reviewed the methodologic aspects of tracer quantification in oncologic patients (4). The authors concluded that the SUV is helpful despite these limitations. Pharmacokinetic quantification is mainly confined to application in a research setting. Indeed, the acquisition of a full dynamic PET scan for $60 \mathrm{~min}$ is difficult for routine purposes. Therefore, shortened acquisition protocols may be helpful in solving this problem.

For tracers such as ${ }^{18} \mathrm{~F}$-FDG, a 2-tissue-compartment model is the most appropriate model to assess the tracer kinetics. It was shown, for example, for colorectal tumors and lung tumors, that follow-up examinations with ${ }^{18} \mathrm{~F}-$ FDG and compartment modeling provide the possibility of predicting the therapeutic effect individually $(5,6)$. However, the use of a 2-tissue-compartment model demands a dynamic PET/CT acquisition for about $60 \mathrm{~min}$, limiting the use of dynamic imaging for routine purposes because of the extended time needed for dynamic and whole-body imaging. Thus, new methods to shorten the dynamic acquisition time and maintain accurate information about the tracer kinetics would be helpful. Besides compartment analysis, the shortened acquisition protocols can also be helpful for the calculation of the global metabolic rate. Visser et al. evaluated shortened dynamic PET with ${ }^{18} \mathrm{~F}-\mathrm{FDG}$ and concluded that a 30-min data acquisition was sufficient to calculate the glucose metabolic rate (7). 
The 2-tissue-compartment model is the standard model to assess the kinetics of ${ }^{18} \mathrm{~F}-\mathrm{FDG}$; therefore, the software for the evaluation of shortened acquisition protocols must provide the compartment parameters for this model, with acceptable accuracy. Usually, an iterative solution of the differential equations is preferred, based on the LevenbergMarquardt algorithm. A 60-min dynamic data acquisition is usually preferred for dynamic studies with ${ }^{18} \mathrm{~F}$-FDG. The use of shorter acquisition times, such as $30 \mathrm{~min}$, may provide false data about the compartment parameters if the standard iterative solution is applied, because only the acquired data (0-30 min) are taken into account for the calculation of the compartment parameters, and data for the 30- to 60-min interval are lacking. Furthermore, the conventional approach of calculating the compartment parameters is generally sensitive for overfitting even with a 0 - to 60-min data acquisition and is therefore user-dependent, thus limiting the reproducibility of the compartment data.

Recently, we developed a software program for the 2tissue-compartment model based on a database and a modified support vector machines (SVM) algorithm (8). The program applies a modified machine-learning algorithm (SVM) to the measured VOI data. The SVM algorithm is a predictive approach; therefore, it is possible to predict the 0 - to 60-min compartment parameters from 0 - to 30-min PET time-activity data, in contrast to the iterative approach, which is confined to the measured time interval. The purpose of this study was to assess the SVM approach for shortened dynamic acquisitions to obtain 60-min compartment data from dynamic acquisitions of 10-30 min. The results of the shortened acquisitions were compared with those results obtained with the standard 0- to 60-min dynamic series, which served as the reference.

\section{MATERIALS AND METHODS}

The study is based on 1,474 time-activity curves obtained from 539 patients in whom dynamic PET studies for 0-60 min were performed. All patients were scheduled for diagnostic purposes to undergo dynamic PET because of a known primary or recurrent tumor. In the cases of recurrent tumors, the patients had undergone previous surgery. All tumor histologies were accepted for this study; the selection was based only on the presence of a malignant lesion. Furthermore, follow-up studies in patients receiving chemotherapy were included. The dynamic series was focused on the region of the tumor, followed by a whole-body scan. We used 28 frames as a standard for the dynamic series $(10 \times 30,5 \times 60,5 \times 120$, and $8 \times$ $300 \mathrm{~s}$ ). Iterative image reconstruction was performed (ordered-subset expectation maximization algorithm), and the reconstructed images were converted to SUV images and transferred to a database for further evaluation. The quantitative assessment was done using multiple VOIs for the tumor, metastatic lesions, normal tissue, and a large vessel, preferentially the descending aorta. A VOI is based on multiple regions of interest positioned over the area of interest on several slices. The time-activity data were obtained by the VOIs by quantifying the radionuclide concentration for each time frame of the series. Partial-volume correction was not applied, because all regions had a diameter of at least $8 \mathrm{~mm}$ and the system recovery is
$85 \%$ for this diameter. The 2-tissue-compartment model uses an input function for the calculation of the compartment parameters. It had been already reported by Ohtake et al. that the input function can be accurately obtained via VOIs (9). Therefore, at least 7 ROIs were placed over the descending aorta to obtain the blood data for ${ }^{18} \mathrm{~F}-\mathrm{FDG}$.

All datasets were evaluated by 2 experienced nuclear medicine specialists, and a 2-tissue-compartment model was applied to the data using the standard iterative curve-fitting procedure. We obtained the following 5 parameters: vB, fractional blood volume, also named vessel density; $k_{1}, k_{2}$ (also referred to as $K_{1}, k_{2}$ ), parameters for the transport of ${ }^{18} \mathrm{~F}-\mathrm{FDG}$ into the cells; and $k_{3}, k_{4}$, parameters for the phosphorylation and dephosphorylation of the intracellular ${ }^{18}$ F-FDG. The influx was calculated from the compartment data using influx $=k_{1} \times k_{3} /\left(k_{2}+k_{3}\right)$. The results of the compartment fitting (vB, $k_{1}-k_{4}$ ) were associated with the corresponding time-activity curves (input curve, target curve) and stored in a database. Thus, each dataset of the database consists of the data from a VOI of the input data, a VOI of the target data, and the resulting kinetic parameters obtained by 2-tissue-compartment fitting using the classic iterative Levenberg-Marquardt algorithm.

The full dynamic datasets for $60 \mathrm{~min}$ and their results regarding the 2-tissue-compartment fit, based on the classic iterative solution, were used as reference for the shortened acquisition protocols and the SVM-based compartment fitting. To generate shortened acquisitions, the 0 - to 60 -min time-activity datasets were reduced to smaller dynamic sequences using the following time intervals: $0-10,0-16,0-20,0-25$, and $0-30 \mathrm{~min}$. Then the shortened dynamic series were evaluated, and a 2-tissue compartment was fitted, now based on the SVM method (8). A 2-step procedure was performed: first the current combination of an input and target curve was compared with the database and a subset of comparable input and target curves were selected from the database of the shortened acquisition data. Then the subset of curves was used together with the measured data to predict the 60-min 2-tissue-compartment results by the modified SVM algorithm.

If the Levenberg-Marquardt algorithm would be applied, for example, to a 0 - to 20-min dynamic series, the algorithm calculates the compartment constants only from this time interval. Because of the limited information compared with a 0 - to 60min series, the compartment parameters are usually different from those obtained from a full dynamic series. In contrast, the modified SVM algorithm predicts the 60-min compartment data results from the shortened acquisition series.

Two groups were made for the data evaluation of the shortened acquisition series. The first group consisted of the different shortened acquisition series and the 60-min uptake value, for example, 0 - to 20-min series and 60-min acquisition. The second group comprised only the shortened acquisition series, for example, 0 - to 20-min series. The grouping was performed to assess whether the 60-min acquisition affected the accuracy of the results. The compartment parameters were calculated for both groups with the SVM approach, using the results of the full dynamic series as reference. Furthermore, the influx was used as an additional variable. Correlation coefficients and squared correlation coefficients were used to assess the accuracy of the prediction.

\section{RESULTS}

The basic statistical data (mean, median, minimum, and maximum) are provided in Supplemental Table 1 (supple- 
mental materials are available online only at http://jnm. snmjournals.org). $K_{1}$ has the highest median values, whereas $k_{3}$ revealed the lowest median values. Mean and median values are comparable, reflecting a symmetric distribution of the data.

The correlation coefficients and squares of the correlation coefficients (variance) are shown in Supplemental Table 2. In Supplemental Table 2, we focused on influx, $\mathrm{vB}, k_{1}$, and $k_{3}$, because these are the most important parameters of the 2-tissue-compartment model. The use of the 60min acquisition in addition to the shortened acquisitions generally resulted in higher correlation coefficients and a higher explained variance preferentially for $k_{3}$. Differences of dynamic PET plus the 60-min data as compared with dynamic PET alone were primarily observed for $k_{3}$ and not for $\mathrm{vB}$ and $k_{1}$.

The square of the correlation coefficients reflects the fractional total variance, which is explained by an existing correlation. If 0.8 (corresponding to $80 \%$ of the total data variance) is chosen as a limit for the squared correlation coefficient, the shortened acquisition protocols for 0-16 up to 0-30 min provide this accuracy for both groups, with and without the 60-min data. Only the series based on 0-10 min has a lower accuracy, with an $r^{2}$ of $0.7430(0-10 \mathrm{~min}$ and 60-min data) and $0.5609(0-10 \mathrm{~min})$ for $k_{3}$ (Supplemental Table 2). However, if a higher limit of $r^{2}(0.9 ; 90 \%$ explained variance) is chosen for the squared correlation coefficient, only the combination of a dynamic PET series with the 60-min acquisition fulfills this limit for the 0- to $30-, 0$ - to $25-$, and 0 - to 20 -min series but not for the 0 - to 16- and 0- to 10-min series. The dynamic PET series alone did not achieve the limit of 0.9 for $r^{2}$ for $k_{3}$ for all time intervals. Therefore, for routine studies a shortened acquisition of 0-20 min, followed by a whole-body acquisition at $60 \mathrm{~min}$, is an alternative to the full dynamic study.

For routine purposes, a shortened acquisition without an additional 60-min acquisition may be primarily of interest. The best results for dynamic PET without the 60-min data were achieved for the $30-\mathrm{min}$ series. $k_{3}$ was the primary limiting parameter with an $r^{2}$ of 0.8862 for the 0 - to 30 -min series. If a limit of 0.8 is chosen for the squared correlation coefficient, the 0 - to 25-, 0 - to 20-, and 0- to 16-min series are acceptable. The variation of the data is demonstrated in Figures 1-3. The parameters influx, vB, $k_{1}$, and $k_{3}$ are shown for the 0- to 10-, 0- to 16-, and 0- to 30-min series (Figs. 1-3). The scatterplots demonstrate that $k_{3}$ is the most critical parameter with the highest statistical noise.

Currently, we use a 30-min dynamic PET/CT study, followed by a whole-body acquisition at $60 \mathrm{~min}$ after the tracer application. The advantage of this protocol is that the dynamic PET examination can be performed with most of the patients holding their arms over their head; this arm positioning is not possible for a full dynamic series of $60 \mathrm{~min}$. Furthermore, the patient can rest or move after the 30-min dynamic PET before the whole-body study. The whole-body study is performed with a 2-min acquisition for each bed position; therefore, only about $12 \mathrm{~min}$ are needed in most of the patients. One example is demonstrated in Figure 4. This patient has a recurrent hepatocellular carcinoma. We performed a 30-min dynamic PET/CT, followed by a whole-body study at $60 \mathrm{~min}$ after injection. Data were evaluated for the dynamic PET/CT and whole-body images for the tumor and blood. After the evaluation of the dynamic PET/CT series by VOIs, the whole-body data are added to the dynamic series by providing the time point of the acquisition and SUV. Then the 2-tissue-compartment model was fitted with the SVM-based program and provided results with high accuracy (Fig. 4A). In contrast, if the standard Levenberg-Marquardt method is applied, we obtain an overfitting for $k_{2}$ that exceeds 1 (Fig. 4B). This result is not acceptable. The $k_{\mathrm{x}}$ values must be within the range of $0-1$, because they reflect the relative amount of ${ }^{18} \mathrm{~F}-\mathrm{FDG}$, which is exchanged with the next or previous compartment. Therefore, the maximum value is 1 , which is associated with a complete exchange of all the ${ }^{18} \mathrm{~F}-\mathrm{FDG}$ with the associated compartment. Overfitting not only deteriorates $k_{\mathrm{x}}$ values, such as $k_{2}$ in this case, but has also an impact on all other $k$ values and vB. Therefore, this method is not stable enough for routine use when inexperienced users apply the algorithm. The problem of overfitting is usually solved by the sequential fitting of $\mathrm{vB}$ and $k_{\mathrm{x}}$ values, but this solution demands an experienced user who has long-term experience with compartment models.

\section{DISCUSSION}

Dynamic data acquisitions provide the possibility of gaining details about ${ }^{18} \mathrm{~F}$-FDG tracer kinetics. The standard method is the acquisition of a dynamic series for $1 \mathrm{~h}$ - the time needed to fit a 2-tissue-compartment model to the dynamic data and calculate the compartment parameters. Several studies have already demonstrated that the kinetic data are superior to single SUV measurements for both tumor diagnostics and therapy management (10-15). It was shown in soft-tissue sarcomas that the quantitative parameters of the ${ }^{18} \mathrm{~F}-\mathrm{FDG}$ kinetics help to predict the grading of the tumor (11). The differentiation of a colorectal tumor from normal colon was quantitatively assessed in a study by Strauss et al. (3). If the full kinetic data were used, only 1 false classification was noted and the overall accuracy was $97.3 \%$.

Comparable results are reported for therapy management studies. In sarcomas, the combination of the mean SUV and $k_{1}$ before and after 1 chemotherapeutic cycle was predictive for therapy outcome, with a correct classification rate of $93.3 \%$ for the nonresponding lesions and $80 \%$ for the responders (12). The quantitative evaluation of soft-tissue sarcomas in 31 patients revealed that the combination of SUV and influx provided an overall accuracy of $83 \%$ for the differentiation of responders from nonrespnders, whereas the use of percentage SUV changes was not helpful, with an accuracy of $58 \%$ (13). Okazumi et al. evaluated 79 patients with sarcomas using dynamic PET (14). The data 
FIGURE 1. Selected scatterplots for dynamic PET series at 0-10 min after tracer injection. Full dynamic series (0-60 min) results are used for reference. $\mathrm{dPET}=$ dynamic PET; inf = influx.
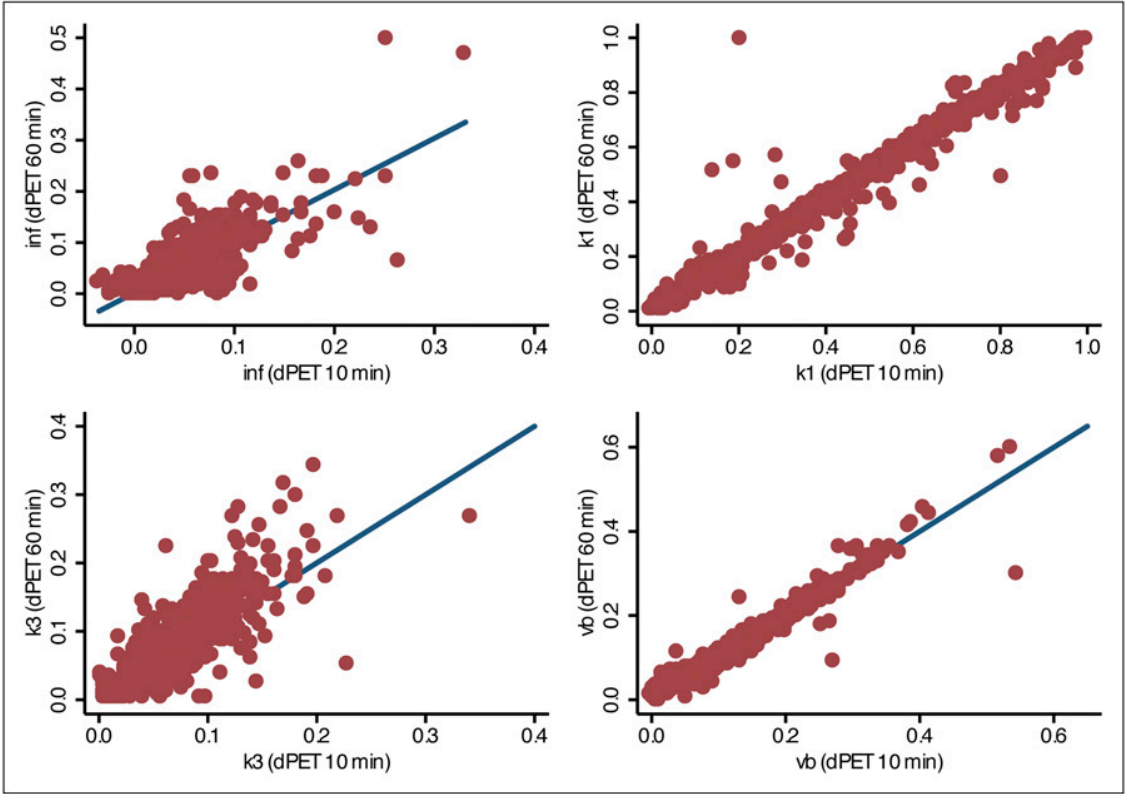

obtained in 71 postoperative patients revealed a sensitivity of $80.85 \%$ and specificity of $87.50 \%$ for the detection of recurrent disease, using $\mathrm{SUV}, \mathrm{vB}, k_{3}$, influx, and fractal dimension as parameters. Kimura et al. evaluated kinetic modeling in patients with gliomas and lymphomas of the central nervous system (15). They concluded that kinetic analysis helps to delineate malignant lesions in the brain. However, more studies are needed to assess the impact of dynamic PET in oncology.

Modeling of tracer kinetics is mainly confined to scientific studies because of the time-demanding procedure and complex evaluation. Protocols with a shortened acquis- ition time may be considered for routine use because of the shortened acquisition time for the dynamic study and faster processing of the data. Therefore, we evaluated if dynamic acquisitions up to 30 min may be helpful to cut down the acquisition time for dynamic studies. However, a key problem with shortened acquisitions is the use of appropriate software to gain accurate information about the tracer kinetics. The conventional approach using the LevenbergMarquardt algorithm for a shortened acquisition protocol, as compared with a full kinetic series, provides different results because the algorithm tries to solve the differential equations for the shortened time interval only. Therefore,
FIGURE 2. Selected scatterplots for dynamic PET series at 0-16 min after tracer injection. Full dynamic series (0-60 min) results are used for reference. $\mathrm{dPET}=$ dynamic PET; inf = influx.
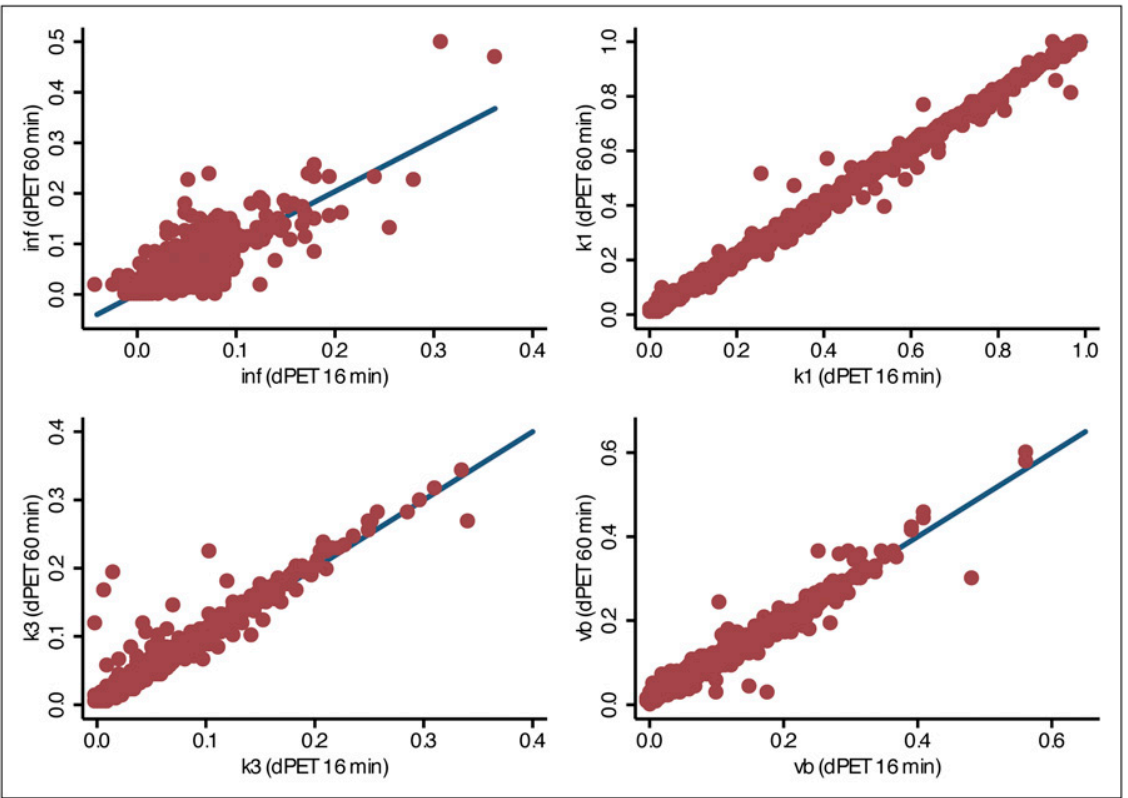

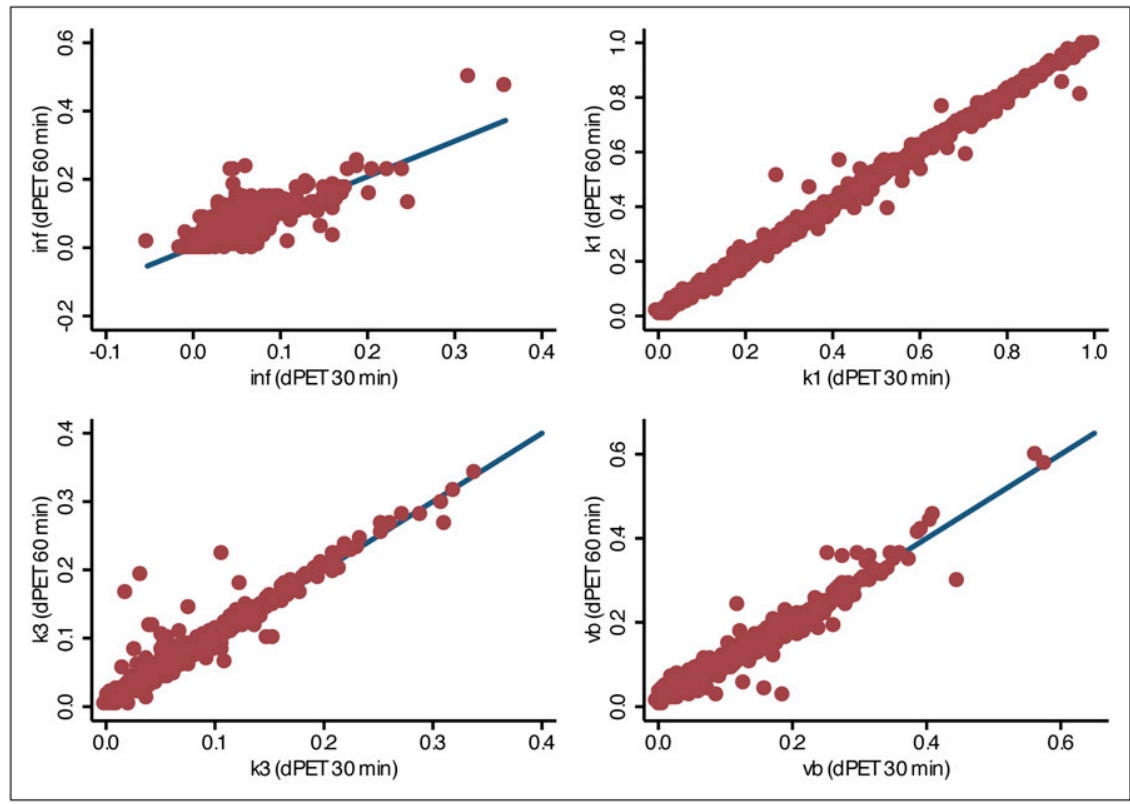

FIGURE 3. Selected scatterplots for dynamic PET series at 0-30 min after tracer injection. Full dynamic series (0-60 min) results are used for reference. $\mathrm{dPET}=$ dynamic PET; inf = influx. predictive, regression-based methods are needed to predict the results of a 60-min series from shortened acquisition data.

We developed a new approach for the calculation of the parameters of the 2-tissue-compartment model based on a modification of the SVM algorithm. The SVM algorithm is a machine-learning method, which is usually applied for data classification (16). The method had been used for the detection of prostate cancer with MRI and the classification of lymph nodes $(17,18)$. Ozer et al. assessed the MRI data of 20 patients with biopsy-proven prostate cancer using different methods for classification analysis. The SVM method provided the highest accuracy and detected more than $80 \%$ of the tumors (17). Sattlecker et al. applied the SVM method for the diagnosis of involved lymph nodes. These authors used different linear and nonlinear kernels for the SVM method and were able to identify $100 \%$ of the independent test data using a radial basis function (18). Jayasurya et al. compared a Bayesian network with the SVM model to predict 2-y survival in 322 lung cancer patients (19). The results demonstrate that the SVM method demands a complete dataset and was accurate for the prediction, whereas the Bayesian network was more helpful if incomplete patient data were available. However, with PET we always have the full dataset, and the problem of limited or missing data does not exist for the dynamic series. We used the SVM algorithm together with a complete database of 1,474 reference datasets for the prediction of the kinetic parameters from the shortened acquisition series. The SVM prediction of the 60-min kinetic parameters from the shortened acquisition series provided, overall, an acceptable accuracy. If a squared correlation coefficient of at least $80 \%$ is accepted, even a single dynamic PET series for $0-16 \mathrm{~min}$ is adequate to predict the kinetic parameters
(Supplemental Table 2). Usually a 60-min whole-body acquisition is performed in oncologic patients. In this case, the combination of a 20-min dynamic PET series with the whole-body data provides an accuracy exceeding $90 \%$, which is acceptable for both routine and scientific PET studies. We currently use at our center a combination of a 30-min dynamic PET series with a whole-body acquisition at $60 \mathrm{~min}$ after tracer injection to the compartment parameters and achieve an accuracy exceeding $95 \%$ regarding the explained variance of the data (Supplemental Table 2).

The data acquisition for $1 \mathrm{~h}$ is a problem for most PET centers because of the limited number of patients who can be studied per day. Furthermore, the data fitting with standard software demands experienced users and may be time consuming. Therefore, simplified methods such as the calculation of the global metabolic rate and the SUV have found use for tracer uptake quantification. However, the SUV has some limitations, and, especially for therapy follow-up studies, we prefer the kinetic analysis to achieve more detailed, accurate information. The SUVs summarize nonmetabolized tracer in the vessels, transported but not metabolized tracer in the tissue, and phosphorylated ${ }^{18} \mathrm{~F}$ FDG. Actually, the phosphorylated ${ }^{18} \mathrm{~F}-\mathrm{FDG}$ is of major importance, especially for therapy-monitoring studies, but this fraction cannot be assessed accurately with the SUV alone. We have used the conventional compartment fitting based on the Levenberg-Marquardt algorithm for several years now. However, the problem of overfitting limits the method for use in routine applications and with inexperienced users. To avoid overfitting, the compartment parameters must be sequentially fitted, which is usually time consuming. In contrast, the modified SVM method is user-independent and provides results based on a predictive approach. One parameter that determines the accuracy of 


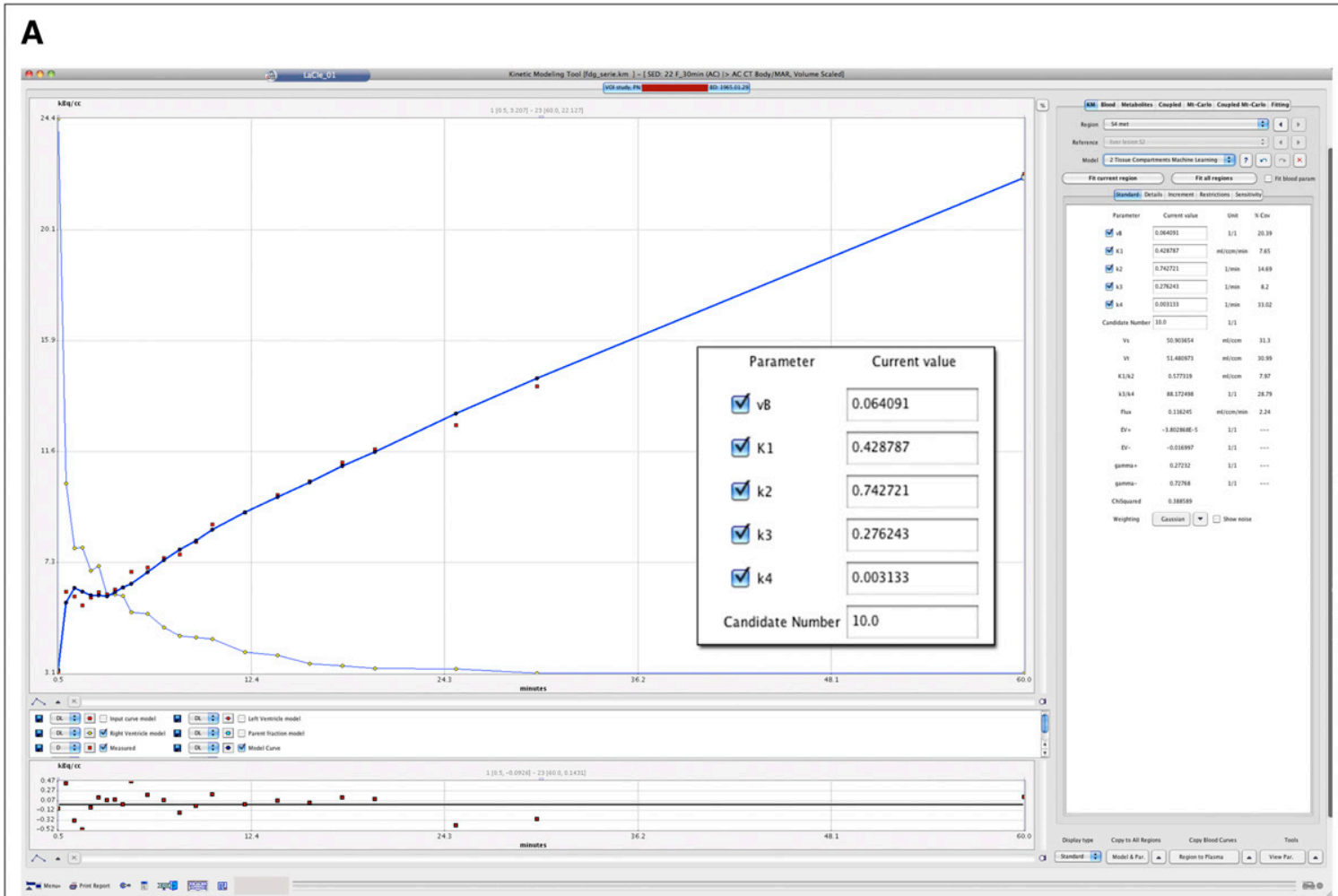

B

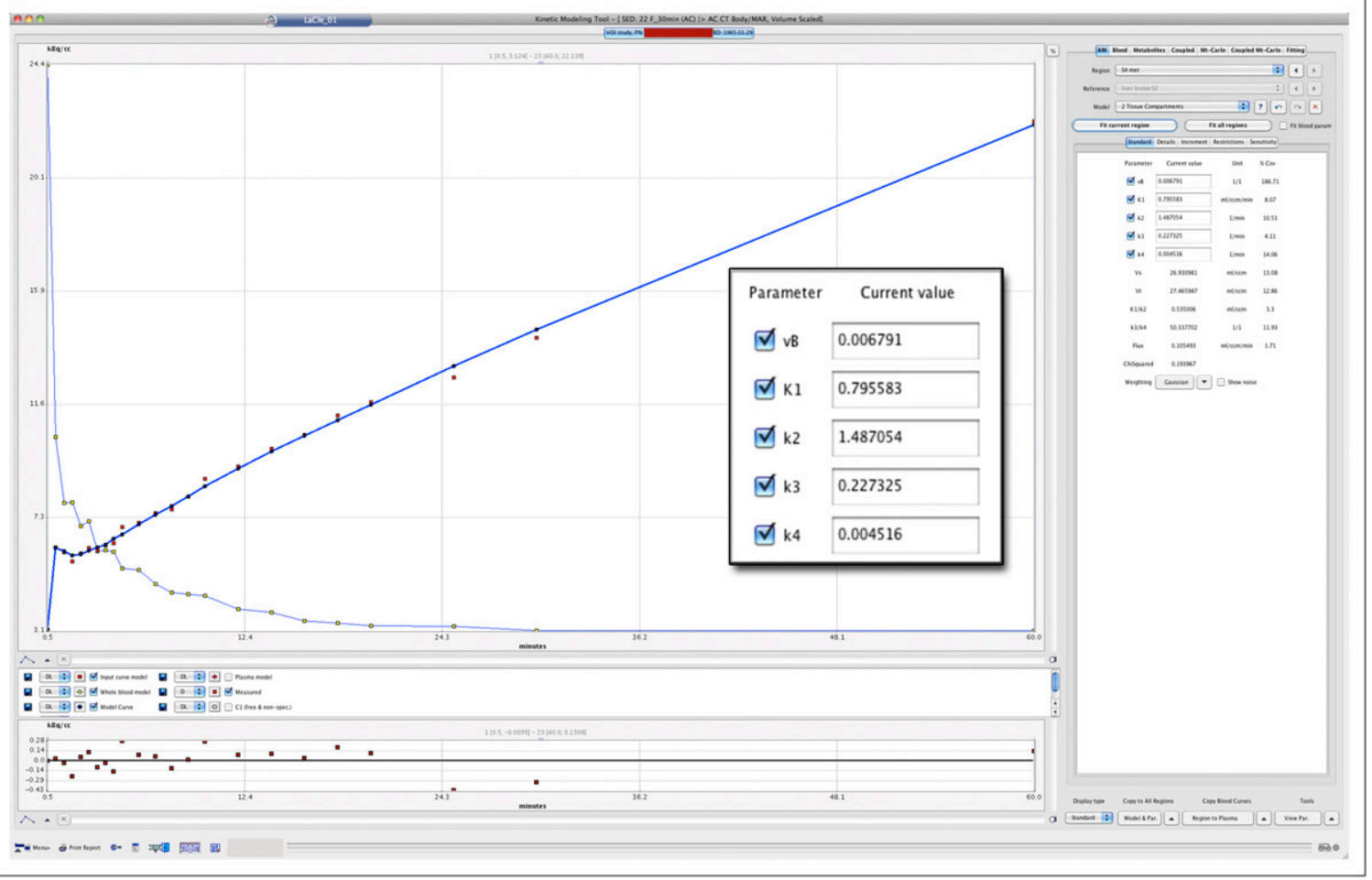

FIGURE 4. (A) Fitting of kinetic model for hepatocellular carcinoma of liver. Input curve was retrieved from abdominal aorta. Target curve is increasing over time because of high metabolism in lesion. Data from 0 to 30 min are obtained from dynamic PET/CT series, and 60-min value is retrieved from whole-body examination. $\chi^{2}$ is 0.389 , demonstrating high accuracy of curve fit based on modified SVM method. These results meet biologic requirements of compartment model $\left(k_{x} \leq 1\right)$ and are obtained without any setting of initial values for model parameters; therefore they are user-independent. (B) A 2-tissue-compartment model fit of data shown in A using Levenberg-Marquardt algorithm. Results demonstrate overfitting of $k_{2}$ with $k_{2}=1.487$. This result is not usable despite good curve fit, because it does not match model assumptions (e.g., $\left.k_{2} \leq 1\right)$. This algorithm is dependent on selection of initial compartment parameters for model. Therefore, it is dependent on experience of user, which may introduce bias. 
the SVM method is the size of the database. Currently, we have nearly 1,500 datasets, and the database is further expanded by adding new dynamic PET studies. However, the current results demonstrate that the dataset of 1,474 time-activity curves is accurate enough to predict the 60-min compartment parameters. We assume that we can improve the accuracy further by adding more time-activity curves to the database. The software used in this study is part of a new software package that we are developing for quantitative dynamic PET/CT studies to provide for its routine use with dynamic $\mathrm{PET} / \mathrm{CT}$.

\section{CONCLUSION}

Short acquisition protocols are helpful in the acquisition of dynamic PET data. The evaluation with modified 2tissue-compartment model software, based on the modified SVM algorithm, provides accurate results for shortened acquisition protocols and avoids overfitting problems.

\section{REFERENCES}

1. Dimitrakopoulou-Strauss A, Strauss L. Quantitative studies using positron emission tomography (PET) for the diagnosis and therapy planning of oncological patients. Hell J Nucl Med. 2006;9:10-21.

2. Strauss LG, Conti PS. The applications of PET in clinical oncology. J Nucl Med. 1991;32:623-648.

3. Strauss LG, Klippel S, Pan L, Schönleben K, Haberkorn U, DimitrakopoulouStrauss A. Assessment of quantitative FDG PET data in primary colorectal tumours: which parameters are important with respect to tumour detection? Eur J Nucl Med Mol Imaging. 2007;34:868-877.

4. Vriens D, Visser EP, de Geus-Oei LF, Oyen WJG. Methodological considerations in quantification of oncological FDG PET studies. Eur J Nucl Med Mol Imaging. 2010;37:1408-1425.

5. Dimitrakopoulou-Strauss A, Hoffmann M, Bergner R, et al. Prediction of shortterm survival in patients with advanced nonsmall cell lung cancer following chemotherapy based on 2-deoxy-2-[F-18]fluoro-D-glucose-positron emission tomography: a feasibility study. Mol Imaging Biol. 2007;9:308-317.
6. Dimitrakopoulou-Strauss A, Strauss LG, Burger C, et al. Prognostic aspects of ${ }^{18}$ F-FDG PET kinetics in patients with metastatic colorectal carcinoma receiving FOLFOX chemotherapy. J Nucl Med. 2004;45:1480-1487.

7. Visser EP, Kienhorst LBE, de Geus-Oei LF, Oyen WJG. Shortened dynamic FDG-PET protocol to determine the glucose metabolic rate in non-small cell lung carcinoma. IEEE Nucl Sci Symp Conf Rec. 2008;4455-4458.

8. Pan L, Cheng C, Dimitrakopoulou-Strauss A, Haberkorn U, Strauss LG. Machine learning based kinetic modeling: a robust and reproducible solution for PET data analysis [abstract]. J Nucl Med. 2009;50(suppl 2):1427P.

9. Ohtake T, Kosaka N, Watanabe T, et al. Noninvasive method to obtain input function for measuring glucose utilization of thoracic and abdominal organs. J Nucl Med. 1991;32:1432-1438.

10. Dimitrakopoulou-Strauss A, Strauss LG, Heichel T, et al. The role of quantitative ${ }^{18} \mathrm{~F}$-FDG PET studies for the differentiation of malignant and benign bone lesions. J Nucl Med. 2002;43:510-518.

11. Dimitrakopoulou-Strauss A, Strauss LG, Schwarzbach M, et al. Dynamic PET ${ }^{18} \mathrm{~F}-\mathrm{FDG}$ studies in patients with primary and recurrent soft-tissue sarcomas: impact on diagnosis and correlation with grading. J Nucl Med. 2001;42:713-720.

12. Dimitrakopoulou-Strauss A, Strauss LG, Egerer G, et al. Prediction of chemotherapy outcome in patients with metastatic soft tissue sarcomas based on dynamic FDG PET (dPET) and a multiparameter analysis. Eur J Nucl Med Mol Imaging. 2010;37:1481-1489.

13. Dimitrakopoulou-Strauss A, Strauss LG, Egerer G, et al. Impact of dynamic ${ }^{18} \mathrm{~F}$ FDG PET on the early prediction of therapy outcome in patients with high-risk soft-tissue sarcomas after neoadjuvant chemotherapy: a feasibility study. $\mathrm{J} \mathrm{Nucl}$ Med. 2010;51:551-558.

14. Okazumi S, Dimitrakopoulou-Strauss A, Schwarzbach MH, Strauss LG. Quantitative, dynamic ${ }^{18} \mathrm{~F}$-FDG-PET for the evaluation of soft tissue sarcomas: relation to differential diagnosis, tumor grading and prediction of prognosis. Hell J Nucl Med. 2009;12:223-228.

15. Kimura N, Yamamoto Y, Kameyama R, Hatakeyma T, Kawai N, Nishiyama Y. Diagnostic value of kinetic analysis using dynamic ${ }^{18} \mathrm{~F}-\mathrm{FDG}-\mathrm{PET}$ in patients with malignant primary brain tumor. Nucl Med Commun. 2009;30:602-609.

16. Chen P-H, Lin C-J, Schölkopf B. A tutorial on v-support vector machines. Appl Stoch Models Bus Ind. 2005;21:111-136.

17. Ozer S, Langer DL, Liu X, et al. Supervised and unsupervised methods for prostate cancer segmentation with multispectral MRI. Med Phys. 2010;37: 1873-1883.

18. Sattlecker M, Bessant C, Smith J, Stone N. Investigation of support vector machines and Raman spectroscopy for lymph node diagnostics. Analyst. 2010;135: 895-901.

19. Jayasurya K, Fung G, Yu S, et al. Comparison of bayesian network and support vector machine models for two-year survival prediction in lung cancer patients treated with radiotherapy. Med Phys. 2010;37:1401-1407. 